\title{
Asymptotic Value of the Probability That the First Order Statistic Is from Null Hypothesis
}

\author{
Iickho Song ${ }^{1}$, Seungwon Lee ${ }^{1}$, So Ryoung Park ${ }^{2}$, Seokho Yoon ${ }^{3 *}$ \\ ${ }^{1}$ Department of Electrical Engineering, Korea Advanced Institute of Science and Technology, Daejeon, South Korea \\ ${ }^{2}$ School of Information, Communications, and Electronics Engineering, The Catholic University of Korea, Bucheon, South Korea \\ ${ }^{3}$ College of Information and Communication Engineering, Sungkyunkwan University, Suwon, South Korea \\ Email: i.song@ieee.org, slee@Sejong.kaist.ac.kr, srpark@catholic.ac.kr, *syoon@skku.edu
}

Received August 31, 2013; revised September 31, 2013; accepted October 8, 2013

Copyright (C) 2013 Iickho Song et al. This is an open access article distributed under the Creative Commons Attribution License, which permits unrestricted use, distribution, and reproduction in any medium, provided the original work is properly cited.

\section{ABSTRACT}

When every element of a random vector $\underline{X}=\left(X_{1}, X_{2}, \cdots, X_{n}\right)$ assumes the cumulative distribution function $F_{0}$ and $F_{1}$ with probability $p$ and $1-p$, respectively, we have shown that the probability $\mathcal{S}_{0}$ that the first order statistic of $\underline{X}$ is originally under $F_{0}$ can be expressed as $\mathcal{S}_{0}=n p \int_{-\infty}^{\infty}\left\{1-(1-p) F_{1}(x)-p F_{0}(x)\right\}^{n-1} \mathrm{~d} F_{0}(x)$. We have also shown that $\lim _{n \rightarrow \infty} \mathcal{S}_{0}=\frac{p}{p+(1-p) \xi}$, where $\xi=\lim _{x \rightarrow \bar{x}} \frac{F_{1}(x)}{F_{0}(x)}$ and $\bar{x}=\max \left(x_{0}, x_{1}\right)$ with $\left[x_{i}, \infty\right]$ the support of $F_{i}(x)$. Applications and implications of the results are discussed in the performance of wideband spectrum sensing schemes.

Keywords: Energy; Order Statistic; Probability; Spectrum Sensing

\section{Introduction}

In wideband spectrum sensing (WSS) of wireless communications with a multiple of receive antennas, the primary goal is to find vacant subbands in a wideband channel composed of a multitude of frequency bands [1-4]. It is beneficial in the WSS to have an accurate estimate of the noise variance. For example, in the detection scheme proposed in [5], estimation of the noise variance in a subband is performed based on the observations in all the subbands. The accuracy of the estimate of the noise variance can be shown to depend on the distributions of the observations under the null and alternative hypotheses. The key parameter in the estimation is the probability that the subband with the lowest energy is under the null hypothesis.

In this paper, we focus on the asymptotic value of the probability and discuss its implications in the performance of WSS schemes.

\section{The Probability}

Let $F_{i}$ be an absolutely continuous cumulative dis-

${ }^{*}$ Corresponding author. tribution function (cdf) for $i=0,1$ and $p$ be a number in the open interval $(0,1)$. Consider a vector $\underline{X}=\left(X_{1}, X_{2}, \cdots, X_{n}\right)$ of independent random variables, each of which assumes the cdf $F_{0}$ and $F_{1}$ with probability $p$ and $1-p$, respectively: On the average, $n p$ random variables of the sample $\underline{X}$ has population cdf $F_{0}$ and the rest has $F_{1}$ as the population cdf. Denote by $\mathcal{S}_{i}=\operatorname{Pr}\left\{X_{(1)} \leftarrow F_{i}\right\}$ the probability that the first order statistic $X_{(1)}$ of $\underline{X}$ is one of the random variables [6,7] having the cdf $F_{i}$ by for $i=0$ and 1 .

Lemma 1. The probability $\mathcal{S}_{i}$ can be expressed as

$$
\mathcal{S}_{0}=n p \int_{-\infty}^{\infty}\left\{1-p F_{0}(x)-(1-p) F_{1}(x)\right\}^{n-1} f_{0}(x) \mathrm{d} x
$$

and

$$
\mathcal{S}_{1}=n(1-p) \int_{-\infty}^{\infty}\left\{1-p F_{0}(x)-(1-p) F_{1}(x)\right\}^{n-1} \cdot f_{1}(x) \mathrm{d} x,
$$

where $f_{i}(x)=\frac{\mathrm{d}}{\mathrm{d} x} F_{i}(x)$ denotes the probability density function (pdf) corresponding to the cdf $F_{i}$ for $i=0,1$.

Proof 1. By $E_{k}$ let us denote the event that the cdf of 
$k$ random variables among the $n$ random variables of $\underline{X}$ is $F_{0}$ and that of the rest $n-k$ random variables is $F_{1}$. Then, since $\underline{X}=\left(X_{1}, X_{2}, \cdots, X_{n}\right)$ is an independent random vector, we easily get [8]

$$
\operatorname{Pr}\left\{E_{k}\right\}={ }_{n} C_{k} p^{k}(1-p)^{n-k} .
$$

We can assume that $X_{j} \sim F_{0}$ for $j=1,2, \cdots, k$ and $X_{j} \sim F_{1}$ for $j=k+1, k+2, \cdots, n$ under $E_{k}$ without loss of generality. Then we easily get

$$
\begin{aligned}
& \operatorname{Pr}\left\{X_{(1)} \leftarrow F_{0} \mid E_{k}\right\}=\sum_{j=1}^{k} \operatorname{Pr}\left\{X_{j}=X_{(1)}, X_{j} \sim F_{0} \mid E_{k}\right\} \\
& =k \operatorname{Pr}\left\{X_{1}=X_{(1)}, X_{1} \sim F_{0} \mid E_{k}\right\} .
\end{aligned}
$$

Denoting the pdf of $X_{i}$ and the joint pdf of $X$ under $E_{k}$ by $f_{X_{i}}\left(x_{i} \mid E_{k}\right)$ and $f_{\underline{X}}\left(\underline{x} \mid E_{k}\right)$, respectively, we have $f_{\underline{X}}\left(\underline{x} \mid E_{k}\right)=\prod_{i=1}^{k} f_{0}\left(x_{i}\right) \prod_{i=k+1}^{n} f_{1}\left(x_{i}\right)$, where $\underline{x}=\left(x_{1}, x_{2}, \cdots, x_{n}\right)$. Therefore,

$$
\begin{aligned}
& \operatorname{Pr}\left\{X_{1}=X_{(1)}, X_{1} \sim F_{0} \mid E_{k}\right\}=\operatorname{Pr}\left\{X_{1}<X_{2}, X_{1}<X_{3}, \cdots, X_{1}<X_{n} \mid E_{k}\right\} \\
& =\int_{-\infty}^{\infty} \int_{x_{2}=x_{1}}^{\infty} \int_{x_{3}=x_{1}}^{\infty} \cdots \int_{x_{n}=x_{1}}^{\infty}\left\{\prod_{i=1}^{k} f_{0}\left(x_{i}\right) \cdot \prod_{i=k+1}^{n} f_{1}\left(x_{i}\right)\right\} \mathrm{d} x_{n} \mathrm{~d} x_{n-1} \cdots \mathrm{d} x_{1}=\int_{-\infty}^{\infty}\left\{1-F_{0}(x)\right\}^{k-1}\left\{1-F_{1}(x)\right\}^{n-k} \cdot f_{0}(x) \mathrm{d} x .
\end{aligned}
$$

Thus, from (4) and (5), we have

$$
\operatorname{Pr}\left\{X_{(1)} \leftarrow F_{0} \mid E_{k}\right\}=k \int_{-\infty}^{\infty}\left\{1-F_{0}(x)\right\}^{k-1}\left\{1-F_{1}(x)\right\}^{n-k} \cdot f_{0}(x) \mathrm{d} x .
$$

Finally, recollecting that $\sum_{k=0}^{n} k_{n} C_{k} x^{k-1}=n(1+x)^{n-1} \quad$ [7,9] and combining (3) and (6), we get (1) as

$$
\begin{aligned}
\mathcal{S}_{0} & =\sum_{k=0}^{n} \operatorname{Pr}\left\{X_{(1)} \leftarrow F_{0} \mid E_{k}\right\} \operatorname{Pr}\left\{E_{k}\right\}=p \int_{-\infty}^{\infty}\left(\sum_{k=0}^{n} k_{n} C_{k}\left[p\left\{1-F_{0}(x)\right\}\right]^{k-1}\left[(1-p)\left\{1-F_{1}(x)\right\}\right]^{n-k}\right) f_{0}(x) \mathrm{d} x \\
& =n p \int_{-\infty}^{\infty}\left\{1-p F_{0}(x)-(1-p) F_{1}(x)\right\}^{n-1} \cdot f_{0}(x) \mathrm{d} x .
\end{aligned}
$$

Following similar steps, we can show (2).

It is straightforward to see that

$$
\begin{aligned}
\mathcal{S}_{0}+\mathcal{S}_{1}= & n \int_{-\infty}^{\infty}\left\{1-p F_{0}(x)-(1-p) F_{1}(x)\right\}^{n-1} \\
& \cdot\left\{p f_{0}(x)+(1-p) f_{1}(x)\right\} \mathrm{d} x=1
\end{aligned}
$$

irrespective of the values of $n$ and $p$, by letting $1-p F_{0}(x)-(1-p) F_{1}(x)=t$, and therefore, $\mathrm{d} t=-\left\{p f_{0}(x)+(1-p) f_{1}(x)\right\} \mathrm{d} x$.

Next, we have

$$
0 \leq 1-p F_{0}(x)-(1-p) F_{1}(x) \leq 1-p F_{0}(x)
$$

since $1-p \geq 0, F_{i}(x) \geq 0$, and $p F_{0}(x)+(1-p) F_{1}(x) \leq \max \left(F_{0}(x), F_{1}(x)\right) \leq 1$. Thus, noting that

$$
\begin{aligned}
\bar{I}_{n}(a, b) & \stackrel{\Delta}{=} n p \int_{a}^{b}\left\{1-p F_{0}(x)\right\}^{n-1} f_{0}(x) \mathrm{d} x \\
& =\left\{1-p F_{0}(a)\right\}^{n}-\left\{1-p F_{0}(b)\right\}^{n},
\end{aligned}
$$

we get $0 \leq \mathcal{S}_{0} \leq \bar{I}_{n}(-\infty, \infty)$ or

$$
0 \leq \mathcal{S}_{0} \leq 1-(1-p)^{n}
$$

from (1) and (9) since $F_{0}(-\infty)=0$ and $F_{0}(\infty)=1$.

\section{Asymptotic Value and Its Implications}

\subsection{Asymptotic Value of $\mathcal{S}_{\mathbf{0}}$}

Let us now obtain the value $\lim _{n \rightarrow \infty} \mathcal{S}_{0}$ more specifically.

Lemma 2. Define

$$
I_{n}(a, b)=n p \int_{a}^{b}\left\{1-p F_{0}(x)-(1-p) F_{1}(x)\right\}^{n-1} \cdot f_{0}(x) \mathrm{d} x,
$$

where $a \leq b$. Then, we have

$$
\lim _{n \rightarrow \infty} I_{n}(a, b)=0 \text { if } F_{0}(a)=F_{0}(b) \text { or } F_{0}(a)>0 .
$$

Proof 2. Recollecting that the pdf $f_{0}(x)$ is nonnegative and (9) holds at any point $x$, it is clear that

$$
0 \leq I_{n}(a, b) \leq \bar{I}_{n}(a, b) .
$$

Now, since

$\lim _{n \rightarrow \infty} \bar{I}_{n}(a, b)= \begin{cases}0, & \text { if } F_{0}(a)=F_{0}(b) \text { or } F_{0}(a)>0, \\ 1, & \text { if } F_{0}(a)=0<F_{0}(b)\end{cases}$

from (10), we immediately have (13) from (14).

Theorem 1. For $i=0,1$, let the support of the $c d f$ $F_{i}(x)$ be $\left[x_{i}, \infty\right]$. Then, we have

$$
\lim _{n \rightarrow \infty} \mathcal{S}_{0}=\frac{p}{p+(1-p) \xi},
$$


where

$$
\xi=\lim _{x \rightarrow \bar{x}} \frac{F_{1}(x)}{F_{0}(x)}
$$

assumes a non-negative value with $\bar{x}=\max \left(x_{0}, x_{1}\right)$.

Remark 1. It is clear from (16) that $\lim \mathcal{S}_{0} \geq p$ if $0 \leq \xi \leq 1, \quad \lim _{n \rightarrow \infty} \mathcal{S}_{0}=p$ if $\xi=1$, and $\lim _{n \rightarrow \infty}^{n \rightarrow \infty} \mathcal{S}_{0} \leq p$ if $\xi \geq 1$.

Remark 2. When $x_{0}<x_{1}$ and $x_{0}>x_{1}$, we have $\xi=0$ and $\xi \rightarrow \infty$, respectively. On the other hand, when $x_{0}=x_{1}$, the value of the non-negative parameter $\xi$ depends on $F_{0}$ and $F_{1}$. Based on this observation, (16) can be expressed as

$$
\lim _{n \rightarrow \infty} \mathcal{S}_{0}= \begin{cases}1, & \text { if } x_{0}<x_{1}, \\ \frac{p}{p+(1-p) \xi}, & \text { if } x_{0}=x_{1}, \\ 0, & \text { if } x_{0}>x_{1} .\end{cases}
$$

Proof 3. (Proof of Theorem ) Assume $x_{0}<x_{1}$, in which case we have $\xi=0$. Since $F_{1}(x)=0$ for all $x \leq x_{1}$, we have

$$
\begin{aligned}
\mathcal{S}_{0}= & n p \int_{-\infty}^{x_{T}}\left\{1-p F_{0}(x)\right\}^{n-1} f_{0}(x) \mathrm{d} x \\
& +n p \int_{x_{T}}^{\infty}\left\{1-p F_{0}(x)-(1-p) F_{1}(x)\right\}^{n-1} f_{0}(x) \mathrm{d} x \\
= & \bar{I}_{n}\left(-\infty, x_{T}\right)+I_{n}\left(x_{T}, \infty\right),
\end{aligned}
$$

where $x_{T}$ is a number in the interval $\left(x_{0}, x_{1}\right]$. Now, we have $\lim \bar{I}_{n}\left(-\infty, x_{T}\right)=1$ from (15) since

$F_{0}(-\infty) \stackrel{n \rightarrow \infty}{=} 0<F_{0}\left(x_{T}\right)$, and we have $\lim I_{n}\left(x_{T}, \infty\right)=0$ from (13) since $F_{0}\left(x_{T}\right)>0$, resulting in $\lim ^{n \rightarrow \infty} \mathcal{S}_{0}=1$ : This result and (8) will after some steps provide us with $\lim \mathcal{S}_{0}=1$, and consequently, $\lim \mathcal{S}_{0}=0$, when $x_{0}>x_{1}$. Here, recollect that $x_{0}>x_{1}$ implies $\xi \rightarrow \infty$.

Next, when $x_{0}=x_{1}$ and $\xi=\lim _{x \rightarrow x_{0}} \frac{F_{1}(x)}{F_{0}(x)}$ are both finite, we can approximate $F_{1}(x)$ as $F_{1}(x) \approx \xi F_{0}(x)$ for a sufficiently small interval $\left[x_{0}, \bar{x}_{0}\right]$ of $x$, where $\bar{x}_{0}>x_{0}$. Then, we can rewrite $\mathcal{S}_{0}$ as

$$
\begin{aligned}
\mathcal{S}_{0}= & n p \int_{x_{0}}^{\bar{x}_{0}}\left\{1-p F_{0}(x)-(1-p) F_{1}(x)\right\}^{n-1} \\
& \cdot f_{0}(x) \mathrm{d} x+I_{n}\left(\bar{x}_{0}, \infty\right) \\
\approx & n p \int_{x_{0}}^{\bar{x}_{0}}\left\{1-\theta F_{0}(x)\right\}^{n-1} f_{0}(x) \mathrm{d} x+I_{n}\left(\bar{x}_{0}, \infty\right) \\
= & \frac{p}{\theta}\left[1-\left\{1-\theta F_{0}\left(\bar{x}_{0}\right)\right\}^{n}\right]+I_{n}\left(\bar{x}_{0}, \infty\right)
\end{aligned}
$$

using (10) since $F_{0}\left(x_{0}\right)=0$, where

$$
\theta=p+(1-p) \xi
$$

is a number larger than $p$. Now, choosing the number $\bar{x}_{0}$ in the open interval $\left(x_{0}, F_{0}^{-1}\left(\frac{1}{\theta}\right)\right)$, we will have $0<1-\theta F_{0}\left(\bar{x}_{0}\right)<1$. Then, we get

$$
\lim _{n \rightarrow \infty} \mathcal{S}_{0}=\frac{p}{p+(1-p) \xi}
$$

from (20) by noting that $\lim _{n \rightarrow \infty} I_{n}\left(\bar{x}_{0}, \infty\right)=0$ from (13) since $F_{0}\left(\bar{x}_{0}\right)>0$.

When $x_{0}=x_{1} \rightarrow-\infty$ and $\xi$ is finite, we can similarly show that (22) holds by employing the approximation $F_{1}(x) \approx \xi F_{0}(x)$ over an interval $\left(-\infty, \bar{x}_{0}\right]$, where $\bar{x}_{0}$ is now a sufficiently small negative,

yet finite, number satisfying $-\infty<\bar{x}_{0}<F_{0}^{-1}\left(\frac{1}{\theta}\right)$.

Finally, following steps similar to those leading to (22) obtained when $x_{0}=x_{1}$ and $\xi$ is finite, we can show that

$$
\lim _{n \rightarrow \infty} \mathcal{S}_{1}=\frac{1-p}{1-p+p \frac{1}{\xi}}=1
$$

quite immediately by symmetry when $x_{0}=x_{1}$ and $\xi$ is infinite: Combining this result with the relation $\mathcal{S}_{0}+\mathcal{S}_{1}=1$ shown in (8), we have $\lim _{n \rightarrow \infty} \mathcal{S}_{0}=0$ when $x_{0}=x_{1}$ and $\xi$ is infinite.

Example 1. Assume that $F_{0}(x)=x u(x)-(x-1) \cdot u(x-1)$ and $F_{1}(x)=\frac{1}{2}\{x u(x)-(x-2) u(x-2)\}$, where $u(x)=1$ for $x \geq 0$ and 0 for $x<0$ is the unit step function. Then

$$
\begin{aligned}
\mathcal{S}_{0} & =n p \int_{0}^{1}\left\{1-p x-(1-p) \frac{x}{2}\right\}^{n-1} \mathrm{~d} x \\
& =\frac{2 p}{1+p}\left\{1-\left(\frac{1-p}{2}\right)^{n}\right\}
\end{aligned}
$$

Thus we have $\lim _{n \rightarrow \infty} \mathcal{S}_{0}=\frac{2 p}{1+p}$, which can also be obtained directly from (16) as $\frac{p}{p+\frac{1}{2}(1-p)}=\frac{2 p}{1+p}$ using $\xi=\lim _{x \rightarrow 0} \frac{F_{1}(x)}{F_{0}(x)}=\frac{1}{2}$, is larger than or equal to $p$.

Example 2. Assume that $f_{0}(x)=\frac{1}{2} \mathrm{e}^{-|x|}$,

$F_{0}(x)=\frac{1}{2} \mathrm{e}^{x} u(-x)+\left(1-\frac{1}{2} \mathrm{e}^{-x}\right) u(x)$, and $F_{1}(x)=F_{0}(x-\mu)$. When $\mu=0$, we will obviously 
have $\mathcal{S}_{0}=p$. Denoting $\mu_{0}=\min (0, \mu)$, we next have

$$
\begin{aligned}
\mathcal{S}_{0}= & n p \int_{-\infty}^{\mu_{0}}\left[1-\left\{p+(1-p) \mathrm{e}^{-\mu}\right\} \frac{\mathrm{e}^{x}}{2}\right]^{n-1} \frac{\mathrm{e}^{x}}{2} \mathrm{~d} x+I_{n}\left(\mu_{0}, \infty\right) \\
= & \frac{p}{p+(1-p) \mathrm{e}^{-\mu}} \cdot\left(1-\left[1-\frac{1}{2}\left\{p+(1-p) \mathrm{e}^{-\mu}\right\} \mathrm{e}^{\mu_{0}}\right]^{n}\right) \\
& +I_{n}\left(\mu_{0}, \infty\right) .
\end{aligned}
$$

Since $0<\frac{1}{2}\left\{p+(1-p) \mathrm{e}^{-\mu}\right\} \mathrm{e}^{\mu_{0}}<1$ from $0<\mathrm{e}^{\mu_{0}} \leq 1$ and $0<\mathrm{e}^{-\mu+\mu_{0}} \leq 1$, we have $\lim _{n \rightarrow \infty} \mathcal{S}_{0}=\frac{p}{p+(1-p) \mathrm{e}^{-\mu}}$ : This value, which can also be obtained directly from (22) and (23) using

$$
\lim _{x \rightarrow-\infty} \frac{F_{1}(x)}{F_{0}(x)}=\lim _{x \rightarrow-\infty} \frac{\mathrm{e}^{x-\mu}}{\mathrm{e}^{x}}=\mathrm{e}^{-\mu},
$$

is larger and smaller than $p$ when $\mu$ is larger and smaller than zero, respectively, and converges to one and zero as $\mu \rightarrow \infty$ and $\mu \rightarrow-\infty$, respectively.

\subsection{Discussion}

In communication systems, we usually have $F_{1}(x)=F_{0}(x-\mu)$, where $F_{0}$ denotes the cdf of noise and $\mu>0$ can be regarded as a measure of the signal to noise ratio (SNR). Now, under the Gaussian, Cauchy, double exponential, and logistic [10] noise environments, from (16) we have $\lim _{n \rightarrow \infty} \mathcal{S}_{0}=1, \quad p, \frac{p}{p+(1-p) \mathrm{e}^{-\mu}}$, and $\frac{p}{p+(1-p) \mathrm{e}^{-\mu}}$, respectively, since $\xi=\lim _{x \rightarrow-\infty} \frac{\exp (2 \mu x)}{\exp \left(\mu^{2}\right)}=0, \quad \xi=\lim _{x \rightarrow-\infty} \frac{x^{2}}{(x-\mu)^{2}}=1$, and $\xi=\lim _{x \rightarrow-\infty} \frac{\mathrm{e}^{x-\mu}}{\mathrm{e}^{x}}=\mathrm{e}^{-\mu}$. These observations imply that

1) we can estimate the noise variance correctly $\left(\lim \mathcal{S}_{0}=1\right)$ by simply increasing the sample size at any positive SNR in the Gaussian case,

2) we can estimate the noise variance correctly by increasing both the sample size and SNR in the doubleexponential and logistic cases, but

3) we cannot estimate the noise variance correctly with probability higher than $p$ by increasing the sample size or SNR in the Cauchy case.

\section{Summary}

We have derived the probability that the first order statistic of a number of independent random variables is originally under the null hypothesis. We have also obtained the asymptotic value of the probability as the sample size tends to infinity, and then we discuss an application and implications of the results in the performance of wideband spectrum sensing schemes.

\section{Acknowledgements}

This study was supported by the National Research Foundation (NRF) of Korea, under Grant 2010-0015175, for which the authors would like to express their thanks.

\section{REFERENCES}

[1] T. Yücek and H. Arslan, “A Survey of Spectrum Sensing Algorithms for Cognitive Radio Applications," IEEE Communications Surveys and Tutorials, Vol. 11, No. 1, 2009, pp. 116-130.

[2] Z. Quan, S. Cui, A. H. Sayed and H. V. Poor, “Optimal Multiband Joint Detection for Spectrum Sensing in Cognitive Radio Networks," IEEE Transactions on Signal Processing, Vol. 57, No. 3, 2009, pp. 1128-1140. http://dx.doi.org/10.1109/TSP.2008.2008540

[3] A. Taherpour, M. Nasiri-Kenari and S. Gazor, "Multiple Antenna Spectrum Sensing in Cognitive Radios," IEEE Transactions on Wireless Communications, Vol. 9, No. 2, 2010, pp. 814-823. http://dx.doi.org/10.1109/TWC.2009.02.090385

[4] P. Paysarvi-Hoseini and N. C. Beaulieu, "Optimal Wideband Spectrum Sensing Framework for Cognitive Radio Systems," IEEE Transactions on Signal Processing, Vol. 59, No. 3, 2011, pp. 1170-1182. http://dx.doi.org/10.1109/TSP.2010.2096220

[5] T. An, H.-K. Min, S. Lee and I. Song, "Likelihood Ratio Test for Wideband Spectrum Sensing," Proceedings of IEEE Pacific Rim Conference on Communications, Computers and Signal Processing, Victoria, 27-29 August 2013.

[6] H. A. David and H. N. Nagaraja, "Order Statistics,” 3rd edition, John Wiley and Sons, New York, 2003. http://dx.doi.org/10.1002/0471722162

[7] I. Song, K. S. Kim, S. R. Park and C. H. Park, "Principles of Random Processes,” Kyobo, Seoul, 2009.

[8] V. K. Rohatgi and A. K. Md. E. Saleh, "An Introduction to Probability and Statistics,” 2nd edition, John Wiley and Sons, New York, 2001.

[9] I. S. Gradshteyn and I. M. Ryzhik, "Table of Integrals, Series, and Products,” Academic, New York, 1980.

[10] J. Hajek, Z. Sidak and P. K. Sen, "Theory of Rank Tests," 2nd edition, Academic, New York, 1999. 